\title{
Colour particle states behaviour in the QCD vacuum
}

\author{
Kuvshinov V.I. ${ }^{1, a}$ and Bagashov E.G. ${ }^{1}$ \\ ${ }^{1}$ Joint Institute for Power and Nuclear Research - Sosny, National Academy of Sciences, \\ 220109 Belarus, Minsk, Acad. Krasin str. 99
}

\begin{abstract}
The results of an interaction of a quantum state of quark with QCD vacuum, where the latter plays a role of environment, could be treated as decoherence. This may have direct implications for the confinement of quarks phenomenon.

The general description and discussion of this process is given. Characteristics from quantum optics and information theory (purity, fidelity, von Neumann entropy) are proposed as means for numerical analysis of the process of interaction of quark colour state with stochastic vacuum.

Problems of stability of colour particles motion and order-chaos transitions are briefly discussed. It is shown that there should be a connection between the properties of QCD stochastic vacuum and Higgs boson mass and self interaction coupling constant.

The behaviour of squeezed and entangled quantum states, the interaction of colour superpositions and multiparticle states with stochastic QCD vacuum is described. It is shown that it leads to a fully mixed quantum state with equal probabilities for different colours. Decoherence rate is found to be proportional to the product of the distance between colour charges and the time during which this interaction has taken place. I.e. such an interaction seems to lead naturally to confinement of quarks.
\end{abstract}

\section{Introduction}

Interactions of some quantum system with the environment can be effectively represented by additional stochastic terms in the Hamiltonian of the system. In this case the averaging with respect to stochastic terms - degrees of freedom of environment - yields the density matrix of the system.

Physically the interactions with the environment result in decoherence and relaxation of quantum superpositions. Information on the initial state of the quantum system is lost after suciently large time [1-4].

Quantum decoherence is the loss of coherence or ordering of the phase angles between the components of a system in a quantum superposition. Decoherence occurs when a system interacts with its environment in a thermodynamically irreversible way. It can be viewed as the loss of information from a system due to the environment (often modeled as a heat bath). Dissipation is a decohering process by which the populations of quantum states are changed due to entanglement with a bath Relaxation usually means the return of a perturbed system into equilibrium. Each relaxation process can be characterized by a relaxation time $\tau$.

ae-mail: v.kuvshinov@sosny.bas-net.by 


\section{Stochastic QCD vacuum}

The model of QCD stochastic vacuum is one of the popular phenomenological models which explains quark confinement (Wilson loop decrease), string tensions and field congurations around static charges [5-7]. In stochastic vacuum only the second-order correlators are important and the others are negligible (they might, however, be important in coherent vacuum) [6], which means Gauss domination. This has been confirmed by lattice calculations [7, 8], although the most important evidence for this is probably Casimir scaling.

In such a situation one assumes that it is possible to calculate vacuum expectation values of gauge invariant quantities as expectation values with respect to some well-behaved stochastic gauge field. It is known that such vacuum provides confining properties, giving rise to QCD strings with constant tension at large distances.

\subsection{QCD vacuum as environment}

We consider QCD stochastic vacuum as the environment for colour quantum particles and average over external QCD stochastic vacuum implementations. Instead of considering nonperturbative dynamics of Yang-Mills fields one introduces external environment and average over its implementations.

As a consequence we obtain: decoherence, relaxation of quantum superpositions, information loss and confinement of colour states phenomenon. It allows us to say that white objects can be obtained as white mixtures of states described by the density matrix as a result of evolution in the QCD stochastic vacuum as environment [9-11].

\section{Colour decoherence}

\subsection{General description}

Consider propagation of heavy spinless colour particle along some fixed path $\gamma$. The amplitude is obtained by parallel transport [9-11]

$$
\begin{gathered}
\partial_{\mu}|\phi\rangle=i \hat{A}_{\mu}|\phi\rangle, \\
|\phi(\gamma)\rangle=\mathcal{P} \exp \left(i \int_{\gamma} d x^{\mu} \hat{A}_{\mu}\right)\left|\phi_{i n}\right\rangle,
\end{gathered}
$$

where $\mathcal{P}$ is the path ordering operator and $\hat{A}_{\mu}$ is the gauge field vector. In order to consider mixed states we introduce the colour density matrix, taking into account both colour particle and QCD stochastic vacuum (environment):

$$
\hat{\rho}(\text { loop, } \gamma \bar{\gamma})=|\phi(\gamma)\rangle\langle\phi(\gamma)|,
$$

Here we average over all implementations of stochastic gauge field (environment degrees of freedom) - and decoherence arises due to interaction with environment.

In the model of QCD stochastic vacuum only expectation values of path ordered exponents over closed paths are defined (in order to keep the gauge invariance). Closed path corresponds to a process in which the particle-antiparticle pair is created, propagates and finally annihilates.

With the help of (1), (2), (3) we can obtain the expression for density matrix $[9,11]$ :

$$
\hat{\rho}(\text { loop }, \gamma \bar{\gamma})=N_{c}^{-1}+\left(\left|\phi_{\text {in }}\right\rangle\left\langle\phi_{\text {in }}\right|-N_{c}^{-1}\right) W_{a d j}(\gamma \bar{\gamma}) .
$$


Here $N_{c}$ is the number of colours.

Colour density matrix in colour neutral stochastic vacuum can be decomposed into the pieces that transform under trivial and adjoint representations $[9,11-13]$

$$
\hat{\rho}_{1}=N_{c}^{-1} \hat{I}+\rho_{1}^{a} \hat{T}_{a}
$$

and Wilson loop in fundamental representation is [6]

$$
W_{\text {fund }}(\text { loop }, \gamma \bar{\gamma})=\left\langle\operatorname{Tr} \mathcal{P} \exp \left(\int_{\text {loop }, \gamma \bar{\gamma}} i \hat{A}_{\mu} d x^{\mu}\right)\right\rangle \text {. }
$$

(Non-abelian Stokes theorem).

In confinement region Wilson loop decays exponentially with the area spanned by loop, so for the rectangular loop spanning the time interval $T$ and distance $R$ we get:

$$
\begin{gathered}
\hat{\rho}(\text { loop }, \gamma \bar{\gamma})=N_{c}^{-1}+\left(\rho_{\text {in }}-\hat{I} N_{c}^{-1}\right) \exp \left(-\sigma_{a d j} R T\right), \\
\hat{\rho}(L: R T \rightarrow \infty)=N_{c}^{-1} \hat{I},
\end{gathered}
$$

where $\hat{I}$ is the unit operator, $\sigma_{a d j}=\sigma_{f u n d} G_{a d j} G_{f u n d}^{-1}$ is the string tension in the adjoint representation, $G_{a d j}, G_{f u n d}$ are the eigenvalues of quadratic Casimir operators. Under Gaussian dominance string tension is

$$
\sigma_{\text {fund }}=\frac{g^{2}}{2} l_{\text {corr }}^{2} F^{2}
$$

$g$ is coupling constant, $l_{\text {corr }}$ correlation length in the QCD stochastic vacuum, $F$ - average of the second cumulant of curvature tensor [7,8].

Here all colour states are mixed with equal probabilities and all information on initial color state is lost. The stronger are the color states connected the stronger their states transform into the white mixture.

\subsection{Decoherence rate, purity, von Neumann entropy}

The decoherence rate of transition from pure colour states to white mixture state can be estimated on the base of purity [3]:

$$
\begin{gathered}
P=\operatorname{Tr} \hat{\rho}^{2} . \\
P=N_{c}^{-1}+\left(1-N_{c}^{-1}\right) \exp \left(-2 \sigma_{\text {fund }} G_{a d j} G_{f u n d}^{-1} R T\right) .
\end{gathered}
$$

When $R T$ tends to $0, P \rightarrow 1$, that corresponds to a pure state. When the composition $R T$ tends to infinity, the purity tends to $N_{c}^{-1}$, which corresponds to the white mixture [11].

The rate of purity decrease is

$$
T_{d e c}^{-1}=-2 \sigma_{\text {fund }} G_{a d j} G_{f u n d}^{-1} R .
$$

Left side of the equation is the inverse characteristic time of decoherence proportional to QCD string tension and distance $R$. It can be inferred from (4) and (11) that the stronger particle-antiparticle pair is coupled by QCD string or the larger is the distance between particle and antiparticle, the quicker the initial state tends to white mixture as a result of interaction with the QCD stochastic vacuum. Thus white states can be obtained as a result of decoherence process. The information of quark colour states is lost in hadrons due to interactions between quarks and confining non-Abelian gauge fields. 
Von Neumann entropy can be used as a measure of the loss of information:

$$
S=-\operatorname{Tr}(\hat{\rho} \ln \hat{\rho}) .
$$

Idempotency of the initial density matrix gives us $S=0$ for the initial state and we will have $S=\ln N_{c}$ for large $R T$. Thus during the interaction with QCD vacuum the entropy increases and the information is being lost.

\section{Colour confinement and instability of colour particle motion}

The stability of quantum motion of the particles is described by quantum fidelity $[14,15]$. The definition of fidelity is similar with Wilson loop definition in QCD [16]. Using the analogy between the theory of gauge fields and the theory of holonomic quantum computation [2, 16, 17], we can define the fidelity of quark (the scalar product of state vectors for perturbed and unperturbated motion) as an integral over the closed loop, with particle traveling from point $x$ to the point $y$ :

$$
f=<\left(\left\langle\phi_{\text {in }}\left|\mathcal{P} \exp \left(\int i \hat{A}_{\mu} d x^{\mu}\right)\right| \phi_{\text {in }}\right\rangle\right)>.
$$

The final expression for the fidelity of the particle moving in the stochastic vacuum is

$$
f=\exp \left(-\frac{1}{2} g^{2} l_{c o r r}^{2} F^{2} S_{\gamma}\right) .
$$

Thus fidelity for colour particle moving along the contour decays exponentially with the surface spanned over the contour, the decay rate being equal to the string tension (9). The motion becomes more and more unstable.

Sometimes fidelity is defined in another way [18-20]:

$$
F(\omega, \tau)=\operatorname{Tr}(\sqrt{\sqrt{\omega} \tau \sqrt{\omega}})
$$

This quantity represents the square root of the probability of transition between the states described by density matrices $\omega$ and $\tau$. If one of the states is pure, then fidelity is equal to the square root of the overlap between the states [21]. Thus sometimes its definition is squared [22]. In our case, for the final state $\hat{\rho}($ loop $)$ and the initial state $\hat{\rho}_{\text {in }}$ we get

$$
\begin{array}{r}
F\left(\hat{\rho}_{\text {in }}, \hat{\rho}(\text { loop })\right)=\operatorname{Tr}\left(\sqrt{\sqrt{\hat{\rho}_{\text {in }}} \hat{\rho}(\text { loop }) \sqrt{\hat{\rho}_{\text {in }}}}\right)= \\
=\operatorname{Tr}\left(\hat{\rho}_{\text {in }} \sqrt{N_{c}^{-1}+\left(1-N_{c}^{-1}\right) W_{\text {adj }}(\gamma)}\right) .
\end{array}
$$

The absence of the second term under the root (when $W_{a d j}(\gamma) \rightarrow 0$ ) returns the value of fidelity $F=N_{c}^{-1 / 2}$. We obtained some non-unit value, which means that the state has changed. And it can be seen how $W_{a d j}(\gamma) \rightarrow 1$ gives us the initial state: $F=1$.

For two random paths in Minkowski space, which are close to each other, the expression for the fidelity is similar, but now the averaging is performed with respect to all random paths which are close enough. Then the final expression is

$$
f=\exp \left(-\frac{1}{2} g^{2} l_{c o r r} \int_{\gamma_{1}} d x^{v} F_{\chi \alpha} \tilde{F}_{v \beta} v^{\chi}\left\langle\delta \chi^{\alpha} \delta \chi^{\beta}\right\rangle\right),
$$


where $\delta \chi$ is the deviation of the path $\gamma_{2}$ from the path $\gamma_{1}, v$ is the four-dimensional velocity and $l_{\text {corr }}$ is the correlation length of perturbation of the particle path expressed in units of world line length.

If unperturbed path is parallel to the time axis in Minkowski space, the particle moves randomly around some point in three dimensional space. The fidelity in this case decays exponentially with time.

Thus we have the connection between confinement and instability of colour particle motion that could be related to possible mechanisms of colour particle confinement.

\section{Order-chaos transition, critical energy of and mass of Higgs boson}

The increasing of instability of motion in the confinement region is also connected with existence of chaotic solutions of Yang-Mills field [9, 23], possible chaos onset [24]. Yang-Mills fields already on classical level show inherent chaotic dynamics and have chaotic solutions [23, 24]. The same is true for quarks.

It was shown that Higgs bosons and its vacuum quantum fluctuations regularize the system and lead to the emergence of order-chaos transition at some critical energy [25, 26]:

$$
E_{c}=\frac{3 \mu^{4}}{64 \pi^{2}} \exp \left(1-\frac{\lambda}{g^{4}}\right)
$$

Here $\mu$ is mass of Higgs boson, $\lambda$ is its self interaction coupling constant, $g$ is coupling constant of gauge and Higgs fields. The expression is valid both for large and small distances. Important here is the value of mass of Higgs boson.

In the region of confinement there exists the point of order-chaos transition where the fidelity decreases exponentially and which is equal to string tension. This connects the properties of stochastic QCD vacuum and Higgs boson mass and self interaction coupling constant.

\section{Squeezed and entangled colour states}

The increasing of instability of motion in the confinement region is also connected with possible quantum entanglement as a probe of confinement and quantum squeezing of colour states [27-30]. The emergence of entagled and squeezed states in QCD becomes possible due to the four-gluon selfinteraction. The three-gluon self-interaction does not lead to the effects [31-33].

The existence of quantum entanglement in Yang-Mills-Higgs theory was considered in [28] on the base of original quasiclassical formalism developed in [27]. The concept of quantum entanglement was found to be very useful as a model-independent characteristic of the structure of the ground state of quantum field theories which exhibit strong long range correlations, most notably lattice spin systems at and near the critical points and the corresponding conformal field theories [34].

Quantum entanglement was also considered as an alternative way to probe the confining properties of large-N gauge theories [29]. Quantum entanglement between the states of static quarks in the vacuum of pure Yang-Mills theory was analyzed in [30]. Hilbert space of physical states of the fields and the charges is endowed with a direct product structure by attaching an infinite Dirac string to each charge. Tracing out the gauge degrees of freedom gives the density matrix which depends on the ratio of Polyakov and Wilson loops spanned on quark world lines [35].

\section{Multiparticle states in QCD vacuum}

Interaction of colour superposition with stochastic QCD vacuum was described in [20]. In its essential parts the process is analogous to the one shown above in Section 3. Let us consider the cases of two and three colour particles. 


\subsection{Two-particle states}

Consider a system of two quarks, denoted as $A$ and $B$. Also consider that there is only two possible colour states for each of them: $|A\rangle,|B\rangle$ and $|\bar{A}\rangle,|\bar{B}\rangle$. Thus $N_{c}=2$.

Here is the list of possible types of states, in which the system may be found (excluding some specific types e.g. as the squeezed states):

- By purity:

- Pure;

- Mixed.

- By separability:

- Separable;

- Non-separable (entangled).

Pure separable system state vector of two quarks can be presented as

$$
|\phi\rangle=|a\rangle|b\rangle,
$$

where $|a\rangle$ and $|b\rangle$ are some states of subsystems $A$ and $B$. Since the system state vector can be factorized (20), we may consider the evolution of each quark independently, using the expressions given in Section 3.

Mixed separable state gives us similar picture, with the only difference being the absence of a state vector. The density matrix of the system will have the form

$$
\rho_{A B}=\rho_{A} \rho_{B},
$$

where density matrices $\rho_{A}$ and $\rho_{B}$ correspond to states of $A$ and $B$ quarks. $\rho_{A}$ and $\rho_{B}$ therefore may also be considered independently with the help of the described mechanism.

We will choose pure non-separable state in the form

$$
|\phi\rangle=\alpha|A\rangle|\bar{B}\rangle+\beta|\bar{A}\rangle|B\rangle .
$$

Density matrix for such a state will have the form

$$
\rho_{A B}=|\alpha|^{2}|A \bar{B}\rangle\left\langle\bar{B} A\left|+\alpha \beta^{*}\right| A \bar{B}\right\rangle\left\langle B \bar{A}\left|+\alpha^{*} \beta\right| \bar{A} B\right\rangle\left\langle\left.\bar{B} A|+| \beta\right|^{2} \mid \bar{A} B\right\rangle\langle B \bar{A}| .
$$

During the evolution and the interaction with vacuum this state will take the form (8), with the exception that it should be raised to a power of $N_{p}=2$, where $N_{p}$ is the number of particles. It can be seen that the entanglement present in (22) vanishes, since the resulting density matrix can be presented in the form $\sum_{i} k_{i} \rho_{i 1} \rho_{i 2}$, where $k_{i}$ is some weight factor, $\rho_{i 1}$ and $\rho_{i 2}$ - some density matrices.

Mixed entangled state is rather difficult to describe. It seems that the easiest way to formally present it in the given case is to artificially suppress (but not entirely) the non-diagonal components of the density matrix - for example by dividing them by 2 . After the interaction with the stochastic vacuum, however, we will again have the state of the form (8). Before the interaction the quantum parameters of the system would be as follows:

The same parameters after the interaction with vacuum (with the state types corresponding to the initial state), also considering fidelity (where the arguments are density matrices of the initial and final states): 
Table 1. Purity and entropy of different states of a two-particle system before the interaction with vacuum

\begin{tabular}{l|llll}
\hline State: & pure separable & mixed separable & pure entangled & mixed entangled \\
\hline $\mathrm{P}$ (purity) & 1 & $\frac{1}{4} \leq \mathrm{P}<1$ & 1 & $\frac{1}{4}<\mathrm{P}<1$ \\
$\mathrm{~S}$ (entropy) & 0 & $0<\mathrm{S} \leq 2 \ln 2$ & 0 & $0<\mathrm{S}<2 \ln 2$ \\
\hline
\end{tabular}

Table 2. General form of purity, entropy and fidelity of different states of a two-particle system after the interaction with vacuum

\begin{tabular}{l|llll}
\hline State: & pure separable & mixed separable & pure entangled & mixed entangled \\
\hline $\mathrm{P}$ (purity) & $\frac{1}{4}$ & $\frac{1}{4}$ & $\frac{1}{4}$ & $\frac{1}{4}$ \\
$\mathrm{~S}$ (entropy) & $2 \ln 2$ & $2 \ln 2$ & $2 \ln 2$ & $2 \ln 2$ \\
$\mathrm{~F}$ (fidelity) & $\frac{1}{2}$ & $\frac{1}{2}<\mathrm{F} \leq 1$ & $\frac{1}{2}$ & $\frac{1}{2}<\mathrm{F}<1$ \\
\hline
\end{tabular}

\subsection{Three-particle states}

Pure separable state vector can be represented as

$$
|\phi\rangle=|a\rangle|b\rangle|c\rangle,
$$

where $|a\rangle,|b\rangle,|c\rangle$ are arbitrary states of subsystems (quarks) $A, B$ and $C$. As in the two-particle case, this situation is analogous to what have already been discussed: if the system's state vector can be factorized, we can discuss the evolution of quarks separately, using expressions derived in the former sections and assuming $N_{c}=3, N_{p}=3$. Of course, the number of dimensions of the density matrix would increase.

In case of mixed separable state we will have similar situation, although without the state vector. Instead of it we will use density matrix to describe the evolution of the system. The density matrix will write as:

$$
\rho_{A B C}=\rho_{A} \rho_{B} \rho_{C},
$$

where the density matrices $\rho_{A}, \rho_{B}, \rho_{C}$ would correspond to the states of quarks $A, B, C$. Each one of these states could be considered independently as well, and its interaction with the stochastic vacuum will be described by the aforementioned mechanism, beginning from the moment of the introduction of density matrix (e.g. (3)). Final state density matrix is determined by the multiplication of density matrices of the final states of the subsystems.

To illustrate the case of pure separable state let us consider the system, analogous to the hadron: here we have three quarks of different colours (and thus $N_{c}=3$ ). We shall symbolically mark these colours by the subscripts $1,2,3$. The three quarks themselves would be marked as $A, B, C$. In this case, for example, the writing $B_{3}$ would mean that the quark $B$ is in the state that is described by the colour 3. And the writing $A_{1} B_{2}$ means that the system is composed of quarks $A$ and $B$ with colour states 1 and 2, respectively. Hadronic structure corresponds to the wave function, such as

$$
|\phi\rangle=a\left|A_{1} B_{2} C_{3}\right\rangle+b\left|A_{1} B_{3} C_{2}\right\rangle+c\left|A_{2} B_{1} C_{3}\right\rangle+d\left|A_{2} B_{3} C_{1}\right\rangle+e\left|A_{3} B_{1} C_{2}\right\rangle+f\left|A_{3} B_{2} C_{1}\right\rangle,
$$

where the complex weight factors $a, b, c, d, e, f$ are normalized as:

$$
|a|^{2}+|b|^{2}+|c|^{2}+|d|^{2}+|e|^{2}+|f|^{2}=1 .
$$

This particular structure of wave function means that the quarks cannot occupy the same colour state. Wave function of antiparticle (with respect to the given hadron) would have the same structure, 
although instead of colour states there would be anti-colour states. So we would consider only the hadron wave function.

During the evolution and interaction with the stochastic vacuum this state will turn into a state that is described by the expression (8), which in the limit $W_{a d j} \rightarrow 0$ (also taking into account $N_{p}=3$ and $N_{c}=3$ ) would give us the expression:

$$
\rho_{A B C}=\operatorname{diag} \frac{1}{27}
$$

As in the two-particle case, the mixed entangled state is impossible to represent in the generalized (analytical) form, since it can have various forms. However, it is still possible to acquire this type of state from the pure entangled state, artificially suppressing the non-diagonal terms. Here also after the interaction with vacuum under the condition $W_{a d j} \rightarrow 0$ we get the state (28).

Before the interaction the parameters of the system would be as follows:

Table 3. Purity and entropy of different states of a three-particle system before the interaction with vacuum

\begin{tabular}{l|llll}
\hline State: & pure separable & mixed separable & pure entangled & mixed entangled \\
\hline $\mathrm{P}$ (purity) & 1 & $\frac{1}{27} \leq \mathrm{P}<1$ & 1 & $\frac{1}{27}<\mathrm{P}<1$ \\
$\mathrm{~S}$ (entropy) & 0 & $0<\mathrm{S} \leq 3 \ln 3$ & 0 & $0<\mathrm{S} \leq 3 \ln 3$ \\
\hline
\end{tabular}

The same parameters after the interaction with vacuum (with the state types corresponding to the initial state), also considering fidelity (where the arguments are density matrices of the initial and final states):

Table 4. General form of purity, entropy and fidelity of different states of a three-particle system after the interaction with vacuum

\begin{tabular}{l|llll}
\hline State: & pure separable & mixed separable & pure entangled & mixed entangled \\
\hline $\mathrm{P}$ (purity) & $\frac{1}{27}$ & $\frac{1}{27}$ & $\frac{1}{27}$ & $\frac{1}{27}$ \\
$\mathrm{~S}$ (entropy) & $3 \ln 3$ & $3 \ln 3$ & $3 \ln 3$ & $3 \ln 3$ \\
$\mathrm{~F}$ (fidelity) & $\frac{1}{\sqrt{27}}$ & $\frac{1}{\sqrt{27}}<\mathrm{F} \leq 1$ & $\frac{1}{\sqrt{27}}$ & $\frac{1}{\sqrt{27}}<\mathrm{F}<1$ \\
\hline
\end{tabular}

\section{Conclusions}

It is productive to consider QCD vacuum as an environment (in the sense of quantum optics) for colour particles. Under certain conditions such an approach leads to confinement of quarks and might serve as an explanation for it.

In the case of of stochastic (not coherent) QCD vacuum (only correlators of the second order are important) in confinement region (Wilson loop decays exponentially) we have decoherence of pure colour states into mixed white states and the onset of instability (chaoticity) of their motion. During this process quantum fidelity and purity decay exponentially (decay rate $=$ QCD string tension).

Dynamics of Yang-Mills fields can be regularized by Higgs fields and quantum fields fluctuations. Critical point of order-chaos transition appears which corresponds to the point, where fidelity and purity drop exponentially. Quantum squeezing and entanglement accompany nonperturbative $\left(A^{4}\right)$ evolution of colour particles in QCD vacuum, confinement, decoherence and instability.

For colour superposition, two and three particle state types (pure separable, mixed separable and nonsepaparable (entangled)) when $R T \rightarrow \infty$ we obtain diagonalization of density matrix, elimination of entanglement, decreasing of purity and fidelity, increasing of von Neumann entropy. 


\section{References}

[1] H. Haken, P. Reineker. The Influence of Exciton Motion on Spin-Resonance. Absorption. Z. Physik. 250, 300 (1972).

[2] P. Reineker. Exciton Dynamics in Molecular Crystals and Aggregates. (Springer-Verlag, Berlin, 1991).

[3] F. Haake. Quantum Signatures of Chaos. (Springer-Verlag, Berlin, 1991).

[4] A. Peres. Quantum Theory: Concepts and Methods. (Kluwer, Dordrecht, 1995).

[5] J. Ambjørn, P. Olesen. On the Formation of a Color Magnetic Quantum Liquid in QCD. Nuclear Physics B. 170, no. 1, 60-78 (1980).

[6] Y. A. Simonov. The Confinement. Uspekhi Fizicheskih Nauk. 4 (1996) (in Russian).

[7] A. D. Giacomo, H. Dosch, V. I. Shevchenko, and Y. A. Simonov, Physics Reports 372, no. 4, 319-368 (2002).

[8] D. S. Kuzmenko, Y. A. Simonov, et al. Vacuum, Confinement and QCD Strings in the Vacuum Correlator Method. Uspekhi Fizicheskih Nauk. 174, no. 1 (2004) (in Russian).

[9] V. Kuvshinov, A. Kuzmin. Gauge Fields and Theory of Deterministic Chaos (Belorussian Science, Minsk, 2006, p. 1-268, in Russian).

[10] V. Kuvshinov, P. Buividovich. White Mixed States in QCD Stochastic Vacuum. Nonlinear Phenomena in Complex Systems. 8, no.3, 313-316 (2005).

[11] V. Kuvshinov, P. Buividovich. Decoherence of Quark Colour States in QCD Vacuum. Acta Physica Polonica B. (Proceedings Supplement), 1, no. 3, 579-582 (2008).

[12] A.O. Barut, R. Raczka, Theory of Group Representations and Applications (World Scientific, Singapore, 1986).

[13] D. Littlewood, The Theory of Group Characters (Clarendon Press, Oxford, 1950).

[14] A. Peres, Stability of Quantum Motion in Chaotic and Regular Systems, Phys. Rev. A30, 16101615 (1984).

[15] T. Prosen, M. Žnidarič, Can quantum chaos enhance the stability of quantum computation? Journal of Physics A: Mathematical and General, L681-L687 (2001).

[16] V. Kuvshinov, A. Kuzmin. Physics Letters A, 316, 391-394 (2003).

[17] V. I. Kuvshinov, P. V. Buividovich. Fidelity and Wilson Loop for Quarks in Confinement Region. Acta Physica Polonica. 36, no. 2, 195-200 (2005).

[18] A. Uhlmann. The "Transition Probability" in the State Space of a *-Algebra. Rep. Math. Phys., 9, 273 (1976).

[19] M. Hübner. Explicit Computation of the Bures Distance for Density Matrices, Phys. Lett. A, 163, 239-242 (1992).

[20] V. I. Kuvshinov, E. G. Bagashov. Evolution of colour superposition in the stochastic QCD vacuum. Nonlinear Phenom. Complex Syst., 16, No. 3, 242-246 (2013).

[21] J. A. Miszczak, Z. Puchala, P. Horodecki, A. Uhlmann, and K. Zyczkowski. Sub- and SuperFidelity as Bounds for Quantum Fidelity, Quantum Information and Computation, 9, no. 1 and 2, 0103-0130 (2009).

[22] R. Jozsa. Fidelity for Mixed Quantum States. J. Mod. Opt. 41 2315-2323 (1994).

[23] G. K. Savvidy. Infrared Instability of the Vacuum State of Gauge Theories and Asymptotic Freedom. Physics Letters B, 71, no. 1, 133-134 (1977).

[24] T. Kawabe et al. Phys. Rev. D. 41, no. 6, pp. 1983-1988 (1990).

[25] V. Kuvshinov, A. Kuzmin. Nonlinear Phenomena in Complex Systems, vol. 2, no.3, 100-104 (1999). 
[26] V. Kuvshinov, A. Kuzmin. J. Nonl. Math. Phys. 9, no.4, 382-388 (2002).

[27] V. Kuvshinov, V. Marmish, V. Shaporov. Theor. Math. Phys., 139, 477-490 (2004).

[28] V. Kuvshinov, V. Marmish. Letters in EPAN v.2, 23 (2005).

[29] L.R. Klebanov, D. Kutasov, A. Murugan. Entanglement as a Probe of Confinement. arXiv:0709.2140v2 [hep-th].

[30] T. Nashioka, T. Takayanagi. JHEP. v.01, 090 (2007).

[31] V. Kuvshinov, V. Shaporov. Acta Physica Polonica B, 30, no. 59, (1999).

[32] V. Kuvshinov, V. Shaparau. Phys. Atom. Nucl., 65, 309-314 (2002).

[33] V. Kuvshinov, V. Shaparau. Acta Physica Polonica B, 35, no. 1, 443-446 (2004).

[34] P. Calabrese and J. Cardy. Int.J.Quant.Inf., 4,429 (2006).

[35] V. I. Kuvshinov, P. V. Buividovich. Nonlinear Phenomena in Complex Systems, 13, no. 2, 149155 (2010). 